\title{
Meta
}

Journal des traducteurs

Translators' Journal

\section{Les mémoires de traduction et la formation universitaire : quelques pistes de réflexion}

\section{Catherine Arrouart}

Volume 48, numéro 3, septembre 2003

Traduction et enseignement

Translation and teaching

URI : https://id.erudit.org/iderudit/007615ar

DOI : https://doi.org/10.7202/007615ar

Aller au sommaire du numéro

Éditeur(s)

Les Presses de l'Université de Montréal

ISSN

0026-0452 (imprimé)

1492-1421 (numérique)

Découvrir la revue

Citer cette note

Arrouart, C. (2003). Les mémoires de traduction et la formation universitaire : quelques pistes de réflexion. Meta, 48(3), 476-479.

https://doi.org/10.7202/007615ar
Résumé de l'article

Les mémoires de traduction font partie de ces outils d'aide à la traduction qui sont de plus en plus utilisés sur le marché de la traduction. Les universités doivent-elles offrir à leurs étudiants une formation sur ces outils? Le cas échéant, de quelle nature devrait être cette formation? 


\section{BLOC-NOTES}

\section{Les mémoires de traduction et \\ la formation universitaire: quelques pistes de réflexion}

\section{RÉSUMÉ}

Les mémoires de traduction font partie de ces outils d'aide à la traduction qui sont de plus en plus utilisés sur le marché de la traduction. Les universités doivent-elles offrir à leurs étudiants une formation sur ces outils? Le cas échéant, de quelle nature devrait être cette formation?

\section{ABSTRACT}

Translation memories are becoming more and more popular in the translation industry. Should universities offer training on these tools to their translation students? And if so, what kind of training should be given?

\section{MOTS-CLÉS/KEYWORDS}

formation universitaire en traduction, outils informatiques, logiciels de mémoire de traduction, localisation, marché canadien

La nécessité d'offrir des cours en informatique dans la formation universitaire en traduction n'est plus à démontrer. Bien des aspects font cependant encore l'objet d'essais, et des questions fondamentales restent sans réponse définitive: faut-il offrir ou imposer une formation en informatique? À quel moment dans le curriculum? Combien d'heures doivent être consacrées à cette matière? Quel contenu enseigner? Quel doit être le rapport entre les heures consacrées à la théorie et à la pratique? Les questions à traiter sont nombreuses; cependant, dans le cadre du présent texte, nous nous limitons à examiner une catégorie d'outils informatiques, les mémoires de traduction, et à proposer des pistes de réflexion concernant leur place dans la formation universitaire en traduction.

\section{Les outils informatiques du langagier: vue d'ensemble}

Il faut, au préalable, faire une distinction entre a) les logiciels d'usage général, c'est-à-dire ceux dont l'usage n'est pas restreint aux langagiers ${ }^{1}$, les logiciels de traitement de texte ou les logiciels de comptabilité par exemple, et b) les logiciels qui sont conçus spécialement pour le langagier, correcteurs et concordanciers entre autres. Les outils informatiques se classent donc en deux catégories: les logiciels de bureautique générale et les logiciels d'aide au langagier, ou outils d'aide à la traduction
(OAT). Le traducteur doit maîtriser les logiciels de bureautique, cela ne fait aucun doute. (Il resterait toutefois à savoir dans quelle mesure.) Ces compétences devraient peut-être même être acquises en dehors du baccalauréat [programme de premier cycle universitaire] au même titre que des connaissances approfondies en langue seconde. Bien sûr, la pratique professionnelle amènera le traducteur à perfectionner sans cesse ses connaissances et ses habiletés mais, en fait, certaines connaissances de base sont le plus souvent déjà une condition préalable à l'embauche.

\section{Les mémoires de traduction}

Les mémoires de traduction (MT), aussi appelées logiciels de mémoire de traduction ou gestionnaires de mémoire de traduction (GMT), translation memories (TM) en anglais, appartiennent aux OAT. La connaissance, d'une part, et la maîtrise, d'autre part, de ces outils sont-elles essentielles au traducteur contemporain? Incombe-t-il à l'université de doter les apprentis traducteurs de connaissances en la matière, et si oui, quelle est la nature de la formation qui devrait idéalement être offerte?

Pour les décrire en quelques mots, les mémoires de traduction sont des bases de données bilingues que le traducteur alimente à mesure qu'il traduit, et dont les éléments (termes, segments de phrases et paragraphes entiers) peuvent être réutilisés par la suite. Les paires de langue forment ce qu'on appelle un bitexte ${ }^{2}$. L'utilisation d'une MT comporte trois avantages principaux: l'uniformisation de la terminologie, surtout dans les projets volumineux dont la traduction est confiée à plusieurs traducteurs; la récupération des phrases ou segments de phrase qui ont déjà été traduits, ce qui permet au traducteur d'éviter la pénible tâche de travailler une deuxième fois les passages difficiles ou de retaper une phrase qui est reprise plusieurs fois; l'archivage systématique des textes, par domaines de spécialité ou par clients, ce qui facilite la consultation ultérieure ou la récupération de matériel traduit. L'utilisation d'une MT est optimisée en présence de textes répétitifs, de nature technique, rédigés dans une langue contrôlée; lorsqu'une terminologie uniforme est utilisée dans plusieurs textes (provenant d'un même client ou appartenant à un même domaine précis) ou lorsque les paires de langues sont réduites (puisque les bases de données constituées sont, jusqu'à présent, bilingues). 


\section{Les MT sur le marché}

Voyons maintenant les MT d'un point de vue extérieur, soit l'usage qui en est fait sur le marché canadien $^{3}$. En fait, le marché exige l'utilisation des MT. Il semble donc que si les donneurs d'ouvrage (soit, au Canada, les gouvernements fédéral et provinciaux, les services de traduction des grandes entreprises et les entreprises de services linguistiques) imposent aux langagiers à qui ils confient des traduction l'utilisation de tel ou tel outil, les étudiants devraient pouvoir inscrire la maitrise de ces outils à la liste de leurs compétences. Or, comme le marché canadien est composé de moins de $20 \%$ de cabinets de traduction et de plus de $80 \%$ de traducteurs indépendants, les traducteurs ne peuvent, dans la majorité des cas, s'en remettre à un employeur pour acquérir de nouvelles compétences.

\section{La place des MT dans la formation universitaire}

Il semble impératif d'offrir aux apprentis traducteurs une formation sur ces outils pour plusieurs raisons: d'abord, pour répondre aux impératifs du marché et ensuite, parce que l'utilisation des mémoires de traduction ne se limite pas à la traduction mais fait partie d'une culture de l'informatique dont l'omniscience se fait de plus en plus sentir, dans tous les aspects des activités professionnelles $\mathrm{du}$ traducteur, plus particulièrement dans un champ d'activités qui connaît une croissance exponentielle, la localisation (dont nous traitons au point 5). Enfin, le dernier motif, et non le moindre, qui justifie la présence d'une formation sur les MT à l'université, c'est le besoin de conscientiser les débutants aux possibilités et aux limites de ces outils, méconnus ou mal connus d'un trop grand nombre de traducteurs. Ce besoin est renforcé par le fait que la plupart des traducteurs canadiens sont en pratique privée et ont seulement des contacts restreints avec leurs pairs: les échanges sur les difficultés qu'ils rencontrent étant limités, certaines situations peuvent avoir des conséquences fâcheuses et parfois irréparables ${ }^{4}$.

\section{Les MT dans le processus de localisation}

De plus en plus d'étudiants se dirigeront vers la localisation au cours des années à venir. Il serait profitable de les doter de connaissances solides des outils qui sont utilisés dans ce domaine. N'attendons pas que sonne le tocsin de la pénurie des spécialistes avant d'inculquer des connaissances qui, si elles peuvent paraître futuristes ou utopiques aujourd'hui, sauront être mises à profit dans quelques années. Il serait intéressant de "prendre le taureau par les cornes» et de s'imposer comme pays d'avant-garde qui forme les étudiants aux besoins que le marché exprimera dans trois ou cinq ans, et de ne pas se faire "damer le pion" par les sociétés étrangères.

\section{Le mirage des MT}

Les MT ne remplaceront jamais les traducteurs. Malgré les craintes que certains peuvent entretenir à l'égard de ces logiciels (il suffit de penser à la vague de panique qu'ont soulevée, dans les années 1970, les chimériques logiciels de traduction automatique), les MT ne font pas concurrence aux traducteurs... Les MT ne traduisent rien, elles répètent! Elles sont en quelque sorte le perroquet du traducteur, un perroquet qui a... une mémoire d'éléphant.

\section{Utilisation abusive des MT}

La MT, ou la base de données plus précisément, a quelque chose de personnel. Elle contient les traductions d'un traducteur. C'est le caractère personnel de la mémoire qui permet d'assurer un usage efficace des MT. Or, dans les grandes sociétés, les MT sont un bien collectif. Tout le monde donne, tout le monde reçoit. Les segments «déjà traduits» sont parfois inutilisables, et il faut les retraduire. Là où ça ne va plus du tout, c'est lorsque le traducteur, en pratique privée, n'est payé que pour les mots qu'il traduit (ceux qui sont déjà traduits dans la mémoire fournie par le client ne comptent pas). Le traducteur doit donc insérer dans les trous (les trous de mémoire!) les termes ou segments de phrases appropriés selon contexte. Certains auteurs ont déjà dénoncé cette aberration (voir Bédard [2000] et Schwab [2001]).

\section{Les enjeux}

La conscientisation des traducteurs aux possibilités et aux limites des MT concerne six éléments ou enjeux dont il est impératif que les traducteurs, chevronnés et débutants, aient connaissance.

a) Rapport entre le gain de rapidité et la perte de temps en apprentissage et utilisation

Si la «machine» est censée faire gagner du temps à son utilisateur, il en va souvent tout autrement dans la réalité. Toutes les heures passées à se familiariser avec le logiciel, à constituer et à nettoyer les bases de données, à régler les bogues, etc., doivent être prises en compte. En un mot, il faut investir le temps juste nécessaire pour faire travailler la machine et non se faire esclave de la machine, sinon l'utilisation de programmes sophistiqués n'est pas défendable.

b) Relation entre la productivité et la qualité Si la MT permet au langagier d'augmenter sa productivité, son utilisation est-elle pour autant synonyme de constance de la qualité? La question mérite réflexion. Le fait d'utiliser une MT (ou tout 
autre logiciel de pointe) n'accroît en rien les compétences linguistiques du langagier. Il faut rester prudent face à la technologie et éviter d'accorder à ses produits une confiance aveugle.

c) Droits d'auteur

Si la question a été posée et reposée, elle n'est toujours pas résolue. Le donneur d'ouvrage a-t-il le droit de réclamer au traducteur indépendant la base de données que ce dernier a constituée au fil de sa traduction? Et à quel prix? Quel usage peutil en faire par la suite? Ces questions demeurent encore sans réponse et suscitent des débats houleux, mais il est primordial que les étudiants sachent au moins qu'elles sont posées.

d) Coût de l'acquisition d'une licence d'utilisation Il faut investir entre plusieurs centaines et quelques milliers de dollars pour obtenir une licence d'utilisation (version personnelle) de l'un des logiciels de MT actuellement offerts sur le marché. Pourtant, tous les outils sont construits autour du même principe et utilisent un même format (TMX, pour Translation Memory Exchange) qui assure la transparence entre les différents produits. Pourquoi alors celui-ci est-il plus cher que celui-là? Qu'offre-t-il de plus? Il faut inviter les étudiants à faire des recherches et à faire preuve d'esprit critique avant d'engager de folles dépenses.

e) Les tarifs: de la poudre aux yeux

Nous avons assisté, au cours des dernières années, à un phénomène pour le moins incroyable. Les traducteurs qui font usage d'une MT peuvent réutiliser des traductions antérieures (qu'ils ont faites eux-mêmes, il ne faut pas se le cacher!) dans la traduction de nouveaux textes. Rien de neuf ici, vraiment... Tout le monde peut, à son gré, faire des recherches dans ses anciens fichiers et s'inspirer des traductions qui s'y trouvent! Quoi qu'il en soit, les donneurs d'ouvrage, conscients de ce fait, ne se sont pas gênés pour offrir des tarifs moins élevés, considérant que les traducteurs gagnent en rapidité. Il faut en informer les finissants, qui s'apprêtent à tenter leur chance sur le marché du travail, afin qu'ils ne tombent pas dans ces pièges.

f) Le risque de démotivation de l'utilisateur compulsif

Relégué au plan de "recycleur de phrases», tel que caricaturé par Bédard (2000), le traducteur débutant peut être désillusionné par ce travail qu'il croyait créatif s'il travaille aveuglément dans une MT.

Bref, il faut à tout prix éviter au traducteur débutant de tomber dans ces pièges. Il importe de le conscientiser aux possibilités et limites des MT afin qu'il soit en mesure d'y jeter un regard critique qui lui permettra d'en faire un usage intelligent.

\section{La nature de la formation universitaire}

La formation universitaire relativement aux mémoires de traduction doit d'abord et avant tout être de nature informative. L'étudiant doit apprendre comment une MT fonctionne (sur quels principes informatiques elle repose), comment on l'utilise (description générale), quelles sont ses possibilités et limites et quels sont les avantages et les inconvénients inhérents à cette catégorie d'OAT.

Ce qui importe, c'est de regarder loin devant soi. La technologie qui sous-tend les MT est vouée à changer et, d'ici cinq ans peut-être, ces logiciels auront été remplacés par d'autres. L'important, c'est d'assurer la pérennité de la formation, de développer chez les étudiants des aptitudes qui leur serviront toujours, qui pourront évoluer avec la technologie, et servir à l'apprentissage de n'importe quel outil: la curiosité, la débrouillardise, l'autodidactisme, et la culture de l'esprit critique.

\section{L'envers du décor}

Offrir un enseignement sur les mémoires de traduction à l'université ne se fait pas «en criant lapin». Trois aspects sont à considérer, en pesant le pour et le contre. Des solutions existent à toutes les difficultés, mais comme les précédents se font rares dans le domaine, il faut parfois user d'imagination et d'audace.

a) La formation des enseignants

Pour transmettre des connaissances, les enseignants doivent d'abord acquérir eux-mêmes ces connaissances. Les OAT sont, de façon générale, nouveaux. Aussi faut-il pouvoir compter sur des professeurs qui soient non seulement perfectionnés en la matière mais surtout intéressés par la technologie en constante évolution. Solution possible: des spécialistes formés par les fabricants de logiciels peuvent offrir une formation de quelques heures à l'université.

b) L'acquisition du matériel Étant donné le prix considérablement élevé des logiciels ainsi que les restrictions budgétaires auxquelles sont soumises les universités canadiennes, il faut trouver d'autres solutions. Par exemple, il est possible d'équiper les laboratoires d'informatique de produits qui sont offerts gratuitement sur Internet (produits à l'essai ou versions Béta, par exemple) ou encore de profiter de prix spéciaux offerts aux étudiants ou de tarifs de groupes.

c) Niveaux de connaissances variables des étudiants Comme les étudiants ont des degrés de connaissances variables en informatique et qu'ils manifestent par ailleurs des intérêts différents à cet égard, la formation pratique sur les OAT en général et sur les mémoires de traduction en particulier pourrait 
être facultative et adaptée aux différents niveaux de connaissances préalables de petits groupes d'étudiants.

\section{Conclusion}

Dans un premier temps, toutefois, une formation théorique, agrémentée d'une démonstration en classe, pourrait être obligatoire pour tous les étudiants, assez tôt dans le curriculum ${ }^{5}$. Au cours de cet exposé général, les points en litige seraient soulevés afin de susciter la curiosité des étudiants et de les amener à réfléchir aux difficultés que pose l'utilisation de ce type de logiciels. Dans un second temps, les étudiants intéressés par le sujet pourraient suivre un cours pratique en laboratoire, dans le cadre duquel ils pourraient faire une utilisation concrète de différents produits. Ce cours pourrait s'étaler sur plusieurs semaines afin que les étudiants réalisent un projet et travaillent à la traduction de plusieurs textes de façon à pouvoir constituer leur première base de données ou mémoire de traduction.

Si les mémoires de traduction ne sont pas l'outil idéal qu'on avait imaginé au début, elles offrent certes des avantages dont les langagiers ont tout intérêt à profiter. Par conséquent, leur présence dans un programme de formation universitaire en traduction s'impose.

\section{Catherine Arrouart Université Laval, Québec, Canada catherine.arrouart@tpsgc.gc.ca}

\section{NOTES}

1. Le terme langagier a été préféré à traducteur, car il englobe, selon le sens que lui donne de Villers (2000), toutes les personnes dont la profession concerne le traitement de la langue, les activités linguistiques. Le présent article traite plus précisément des traducteurs que des autres langagiers, mais tout traducteur est, à ses heures, terminologue et rédacteur.

2. La paternité du terme est généralement attribuée à Brian Harris, cité dans L’Homme (1999: 22).

3. Il s'agit d'un aperçu sommaire de la réalité au Canada. Pour plus d'information à ce sujet, voir le Rapport final du Comité sectoriel de l'industrie canadienne de la traduction (1999), et Shadbolt (2002).

4. Le fait que des personnes acceptent de travailler par exemple, à des tarifs moindres lorsqu'ils utilisent une MT, contribue à "casser» les prix.

5. Les étudiants, peu importe leur niveau d'études, devraient avoir droit à la formation dont il est ici question. Les étudiants de maîtrise qui arrivent d'un autre domaine d'études n'ont pas vraiment de notion de la réalité du marché et sont vulnérables aux pièges que nous avons décrits.

\section{RÉFÉRENCES}

BÉDARD, C. (2000): «Mémoire de traduction cherche traducteur de phrases", Traduire, no 186, p. 4149.

De Villers, M.-É. (2000): Multidictionnaire de la langue française, $3^{\mathrm{e}}$ édition, Montréal, Québec Amérique.

L'Hомме, M.-C. (1999): Introduction à la traductique, Linguatech éditeur inc., Brossard (Québec).

Comité sectoriel de l'industrie canadienne de la traduction (1999): L'industrie canadienne de la traduction: stratégie de développement des ressources humaines et d'exportation, Rapport final du CSICT.

Shadbolt, D. (2002): «The Translation Industry in Canada", Multilingual Computing and Technology, 46, 13-2, pp. 30-34.

Schwab, W. (2001): "Translation Memories in Transition », Circuit, n ${ }^{\circ} 71$, p. 30. 